Supporting Information

\title{
Fluorescent Polymer Dot-Based Multicolor Stimulated Emission Depletion Nanoscopy Using a Single Laser Beam Pair for Cellular Tracking
}

Yayun Wu, ${ }^{1,2}$ Hefei Ruan, ${ }^{1}$ Zaizai Dong, ${ }^{1}$ Rong Zhao,,${ }^{1,2}$ Jianqiang Yu, ${ }^{1}$ Xiaojun Tang, ${ }^{1}$ Xiaolong Kou, ${ }^{1}$ Xing Zhang, ${ }^{1}$ Manchen Wu, ${ }^{1,2}$ Fang Luo, ${ }^{1,2}$ Jinghe Yuan $^{1 *}$ and Xiaohong Fang ${ }^{1,2,3 *}$

1. Key Laboratory of Molecular Nanostructure and Nanotechnology, CAS

Research/Education Center for Excellence in Molecular Sciences, Institute of Chemistry, Chinese Academy of Sciences, Beijing 100190, China

2. University of Chinese Academy of Sciences, Beijing 100049, China

3. Institute of Cancer and Basic Medicine, Chinese Academy of Sciences, Hangzhou, Zhejiang 310022, China

\section{Table of Contents:}

1. Figure S1. Typical TEM image of PDFDP Pdots.......S3

2. Figure S2. Long-term colloidal stability of Pdots.......S3

3. Figure S3. Zeta potentials of Pdots.......S4

4. Figure S4. Typical intensity profiles of Pdots......S4

5. Figure S5. Cross section of Pdots. .....S5

6. Figure S6. Colocalization of Pdots with lysosomes.......S5

7. Figure S7. Immunofluorescence imaging of Pdots. .....S6

8. Figure S8. Labeling density of Pdots compared with organic dyes......S6

9. Figure S9. STED imaging of Pdots labeled CD44 on membrane.......S7

10. Figure S10. Linear unmixing of crosstalked images.......S8

11. Figure S11. Photostability of Pdots compared with organic dye......S9

12. Figure S12. Photostability of Pdots in dual-color STED nanoscopy. .....S9

13. Figure S13. Singlet oxygen generation efficiency of Pdots. $\cdots . . . \mathrm{S} 10$

14. Figure S14. Phototoxicity of Pdots after laser irradiation. .....S10

15. Figure S15. Endocytosis pathway determination for the Pdots. .....S11

16. Figure S16. Labeling of clathrin-coated and caveolae vesicles......S12

17. Figure S17. Endocytosis of CNPPV and PDFDP-Tf Pdots. .....S12

18. Figure S18. Dual-color STED imaging of endosomes labeled by Pdots......S13

19. Figure S19. Imaging CNPPV Pdots in one vesicle in theory......S13 
20. Figure S20. Slop of long-term photobleaching of Pdots. .....S13

21. Figure S21. Raw data of figure $5 \mathrm{c}$ in the manuscript......S14

22. Figure S22. Inhibiting endosome interactions with wortmannin......S14

23. References.......S15 


\section{Supplementary Figures:}

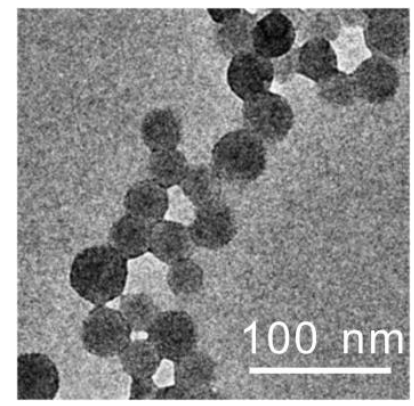

Figure S1. A typical high resolution TEM image of PDFDP Pdots.

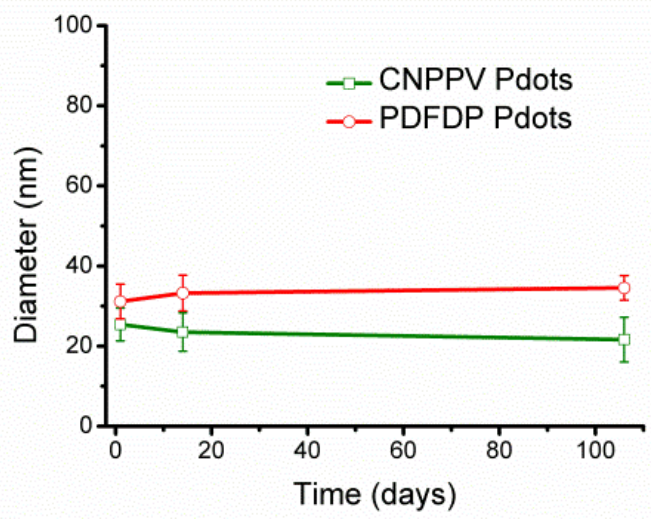

Figure S2. Long-term colloidal stability of CNPPV and PDFDP Pdots for 106 days. After storage for more than 3 months in pure water, the Pdots exhibited good aqueous stability without increasement of sizes or any visible aggregates. 


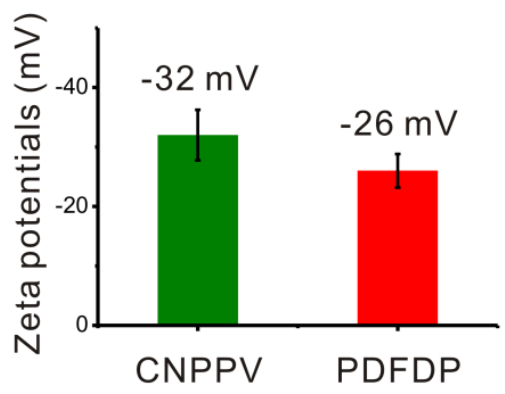

Figure S3. Zeta potentials of CNPPV and PDFDP Pdots were -32 and $-26 \mathrm{mV}$, respectively. Negative charges of nanoparticles indicated the formation of carboxyl groups on the Pdots. Since the two types of semiconducting polymers have different molecular weights and rigidities, their mixtures with PSMA polymers were hydrolyzed to form nanoparticles with different sizes and probably different surface properties (e.g. density of carboxyl groups), thus different zeta potentials.

a

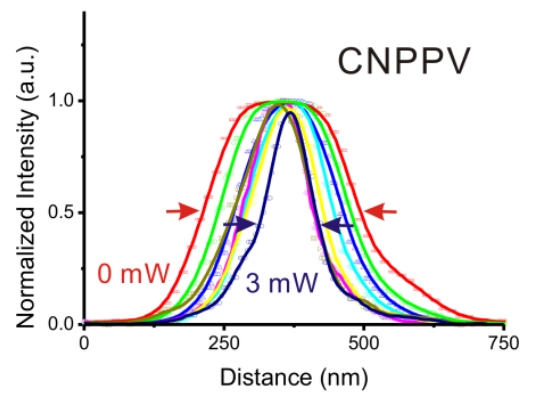

b

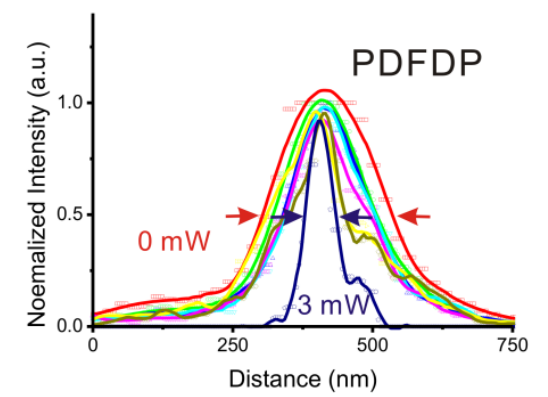

Figure S4. Typical intensity profiles of single (a) CNPPV and (b) PDFDP nanoparticles with the increase of depletion laser power from 0 to $3 \mathrm{~mW}\left(0 \sim 10 \mathrm{MW} / \mathrm{cm}^{2}\right)$. The profiles of single particles became increasingly sharp and the spatial resolutions of two kinds of Pdots were effectively enhanced. 


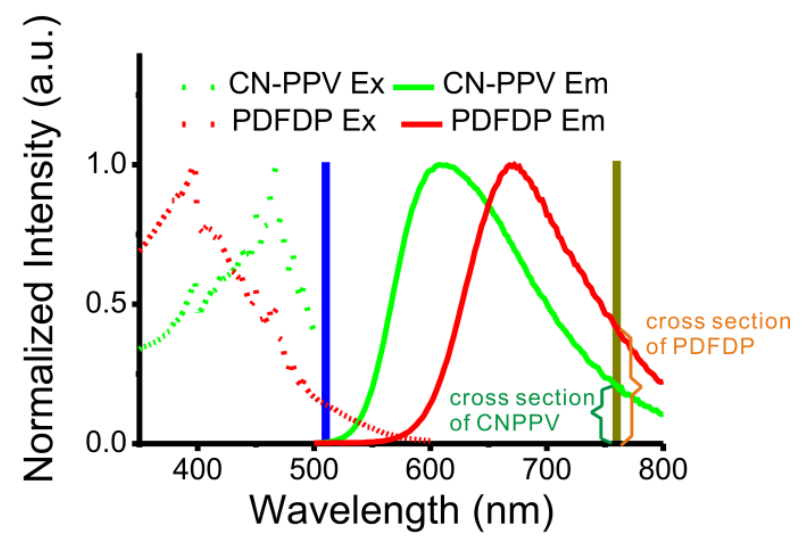

Figure S5. Cross section of PDFDP Pdots was larger than CNPPV Pdots for stimulated emission, thus PDFDP Pdots were more efficiently depleted and exhibit higher resolution.

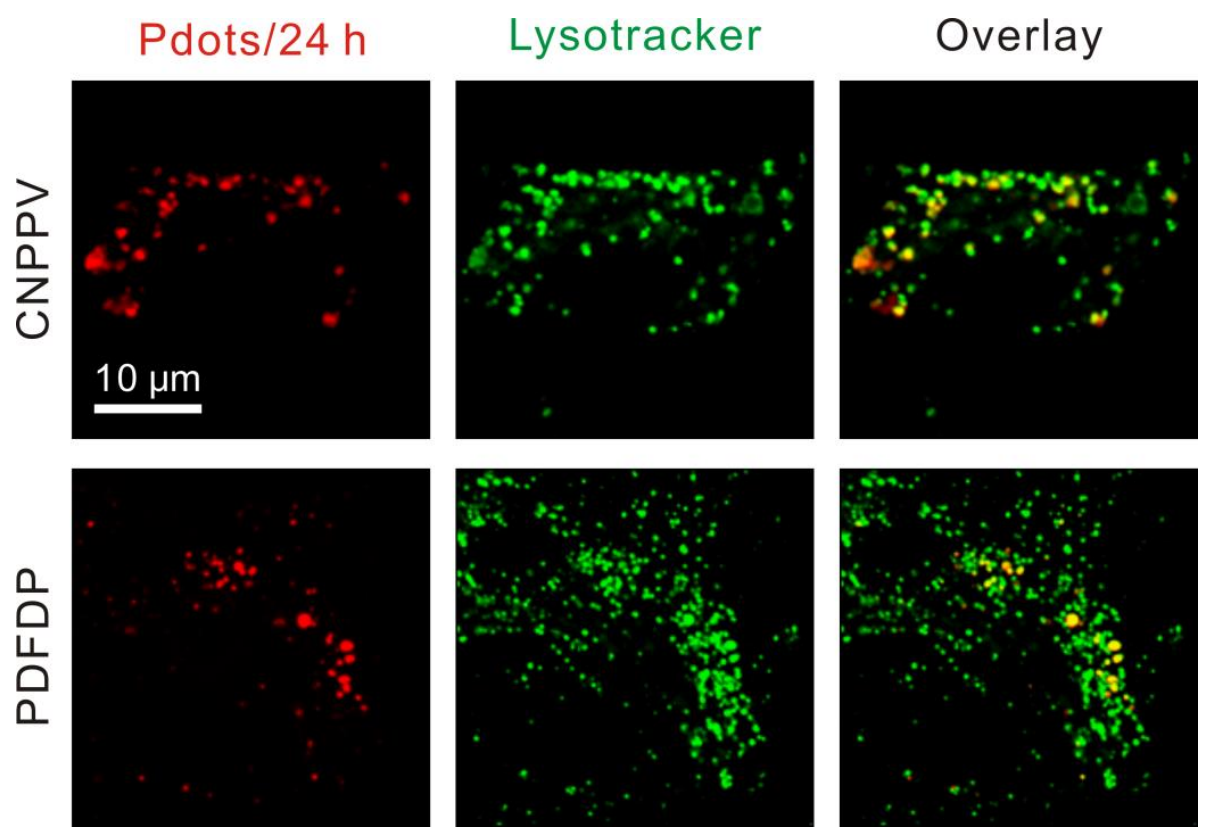

Figure S6. Colocalization of Pdots with lysosomes. After incubating with Hela cells for 24 hours, CNPPV and PDFDP Pdots were localized in lysosomes. So Pdots were able to specifically label lysosomes in cells after 24 hour-incubation. 


\section{a}
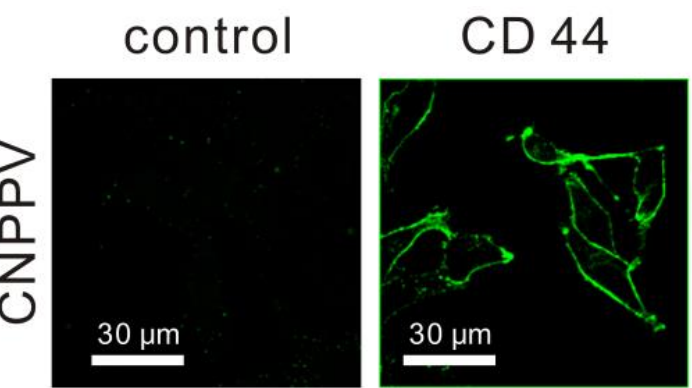

Tubulin

b
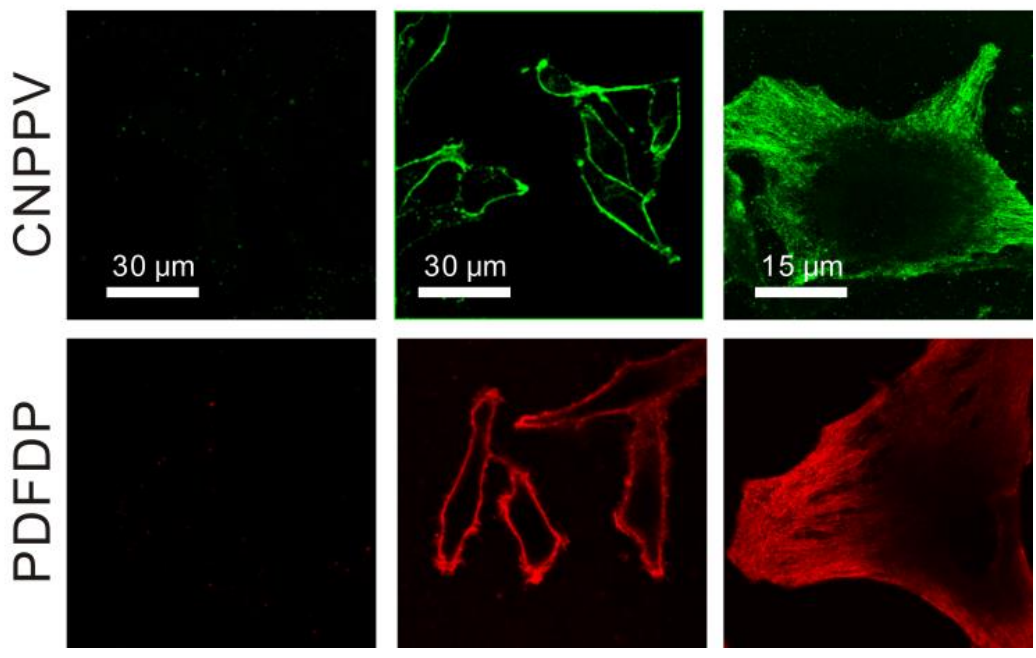

Figure S7. Immunofluorescence imaging of CD44 and tubulin labeled by (a) CNPPV and (b) PDFDP Pdots. With the addition of biotinlated CD44 primary antibody or tubulin primary antibody and then biotinlated secondary antibody, streptavidin conjugated Pdots, cellular samples were specifically labeled. Without CD44 primary antibody or tubulin primary antibody in the control experiment, only streptavidin conjugated Pdots were unable to specifically label cellular targets. Thus no obvious signals were observed in the control images. The results verified the specificity of our labeling methods.

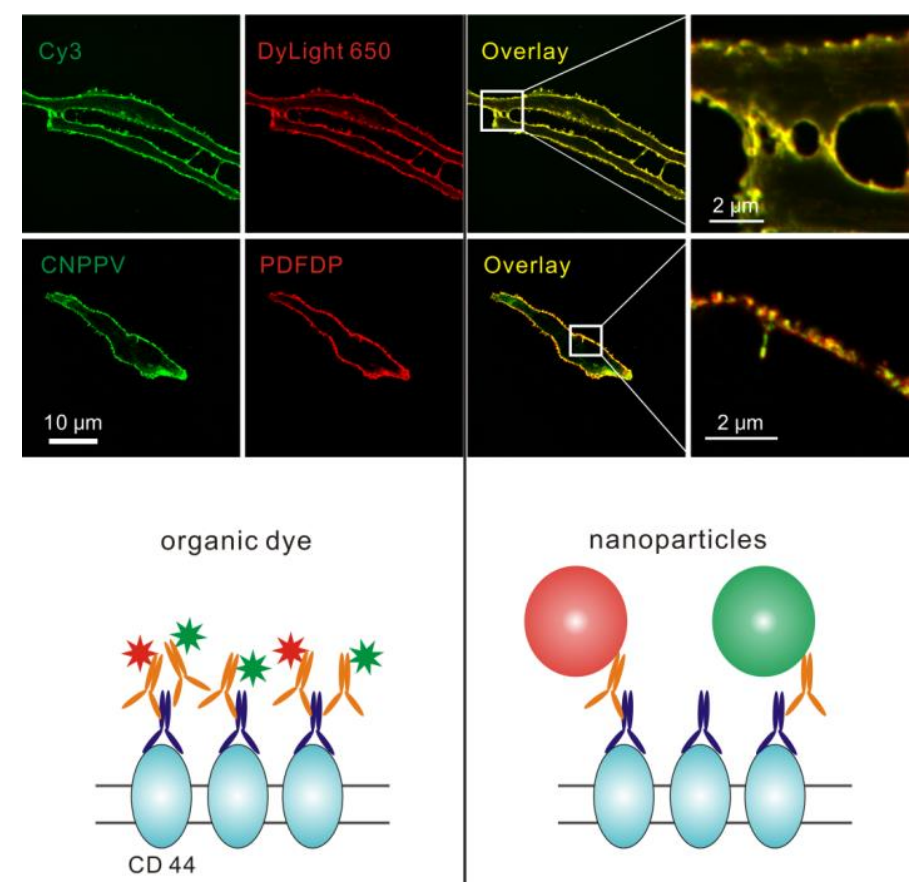

Figure S8. CD44 on the cell membrane was labeled by a mixture of Cy3/DyLight 650 dye molecules and two colors were completely colocalized in confocal images. In contrast, CNPPV and PDFDP Pdots mixtures labeled CD44 were not as well colocalized as organic dyes due to relatively low labeling density. 

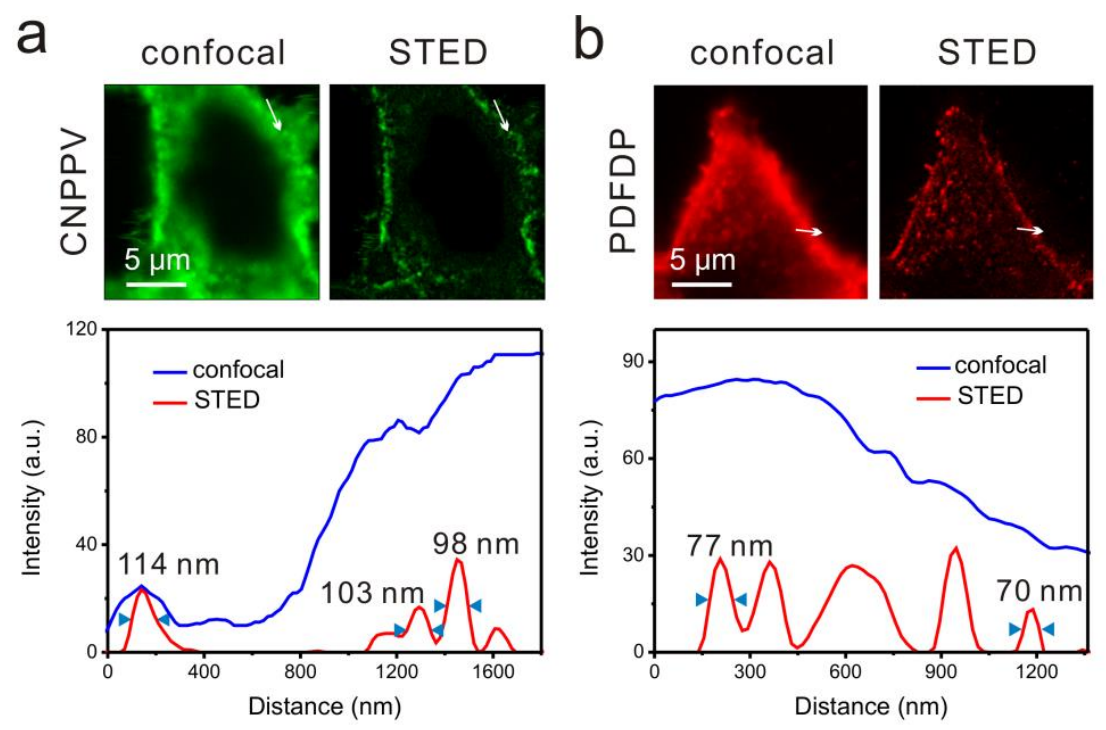

Figure S9. Super-resolution imaging of CNPPV and PDFDP Pdots labeled CD44 on cellular membrane. 
a

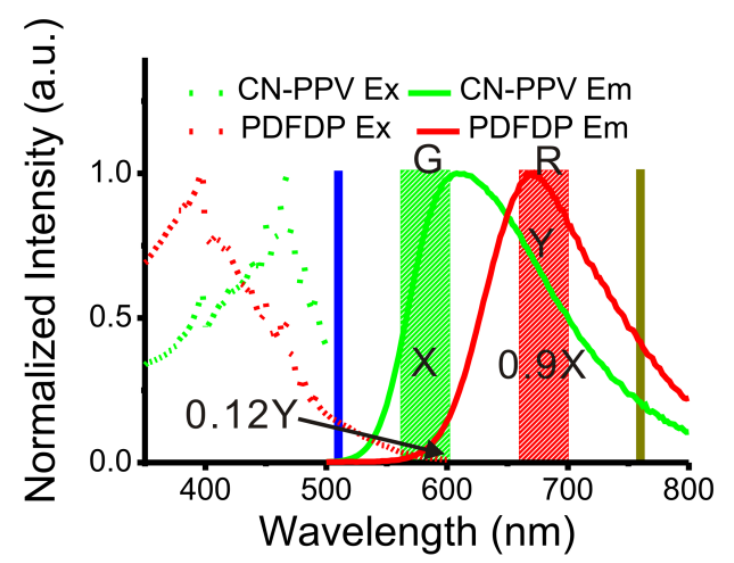

b

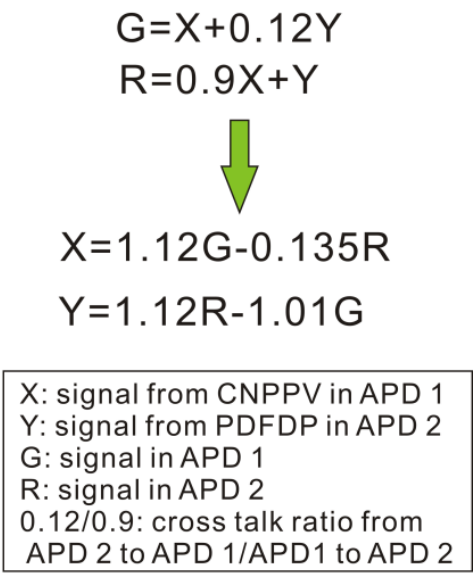

C
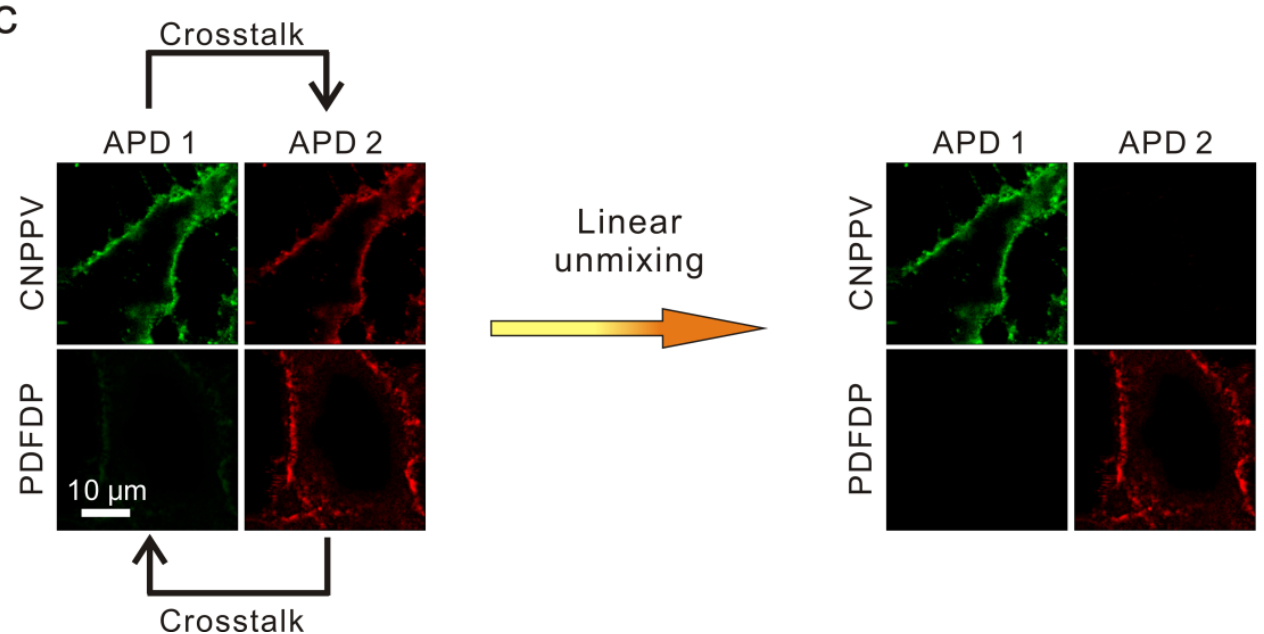

Figure S10. Linear unmixing of crosstalked images. (a) Crosstalk of two channels (green channel: signal from APD 1; red channel: signal from APD 2) was obvious due to the overlap of emission spectra of CNPPV and PDFDP Pdots. (b) Signal in green channel is composed of signal from CNPPV Pdots and crosstalked signal from PDFDP Pdots, and as same as the red channel. Thus, signal of individual Pdots is unmixed by equations in (b). (c) Crosstalk between two detection channels in STED imaging was eliminated by linear unmixing. Depletion power: $10 \mathrm{MW} / \mathrm{cm}^{2}$; pixel dwell time: $1 \mathrm{~ms}$; the field of view: $30 \times 30 \mu \mathrm{m}^{2} ; 200 \times 200$ pixels.

We have calculated the crosstalk as the ratio of fluorescence intensity from the two channels, that is $\mathrm{Fl}_{\mathrm{APD} 2} / \mathrm{Fl}_{\mathrm{APD} 1}(\sim 90 \%)$ for CNPPV Pdots and $\mathrm{Fl}_{\mathrm{APD} 1} / \mathrm{Fl}_{\mathrm{APD} 2}(\sim 12 \%)$ for PDFDP Pdots. So if the signal of CNPPV Pdots in APD1 is $X$, the crosstalked signal in APD2 is $90 \% \mathrm{X}(0.9 \mathrm{X})$. Similarly, the crosstalked signal of PDFDP Pdots (set as Y in APD2) in APD1 is $12 \% \mathrm{Y}$. The total signal in APD1 (G, green) is the sum of $\mathrm{X}$ and $0.12 \mathrm{Y}$, and the signal in APD2 is the sum of $0.9 \mathrm{X}$ and $\mathrm{Y}$. After calculating the above two linear equations in two unknowns, the sigals of Pdots ( $X$ and $Y$ ) without crosstalk can be obtained after calculation of raw crosstalked images according to equations in figureS10b. 


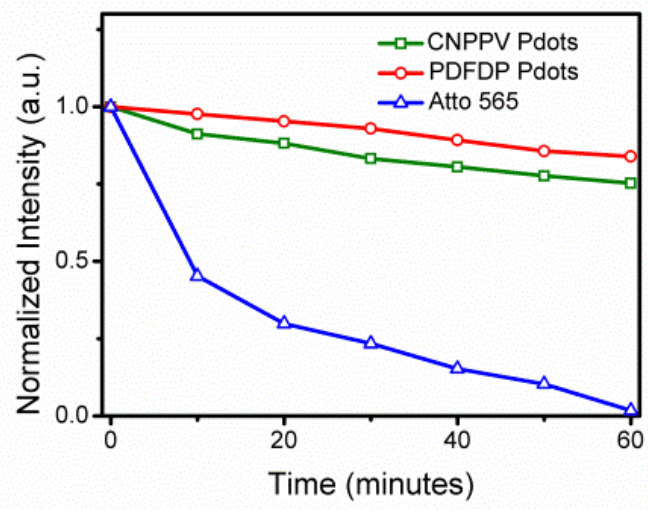

Figure S11. Photostability of CNPPV and PDFDP Pdots compared with organic dye Atto 565. CD44 on cellular membrane were labeled with CNPPV/PDFDP Pdots and Atto 565 organic dyes, respectively. Then the samples were continuously irradiated with STED laser beams for 1 hours and STED images were acquired under same conditions during irradiation period. The intensity changes were recorded and analyzed with Image $\mathrm{J}$ software.

a

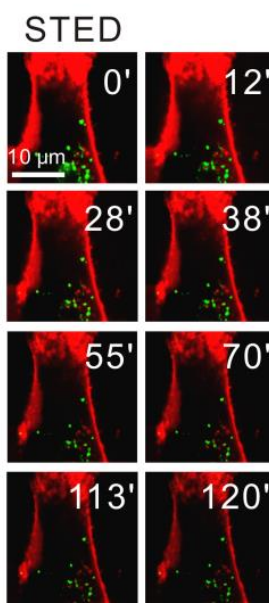

b
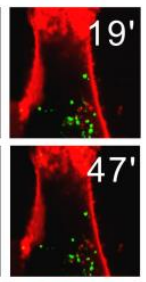

90'
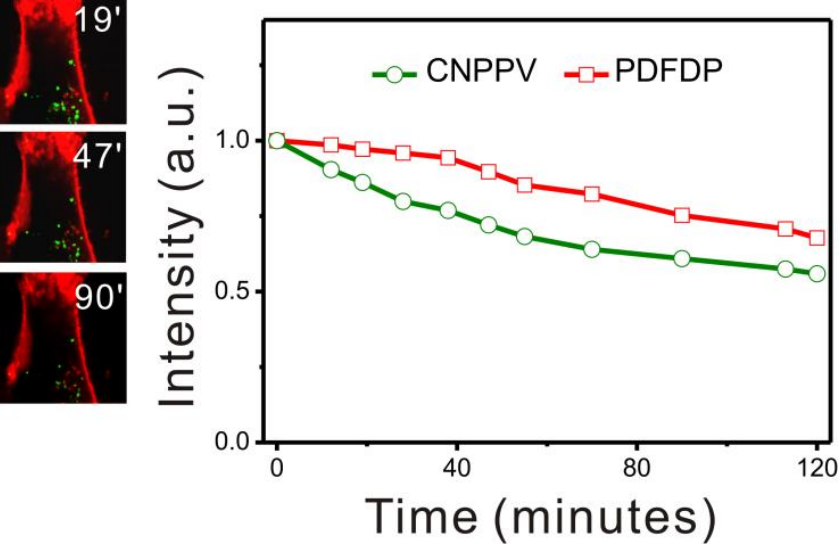

Figure S12. Photostability of CNPPV and PDFDP Pdots in dual-color STED nanoscopy. (a) Continuous long-term STED imaging of CNPPV and PDFDP Pdots for 120 minutes with $10 \mathrm{MW} / \mathrm{cm}^{2}$ depletion power. The CNPPV Pdots labeled lysosomes and the PDFDP Pdots labeled CD44 in a Hela cell. (b) Continuous photobleaching curves of CNPPV and PDFDP Pdots in (a). 


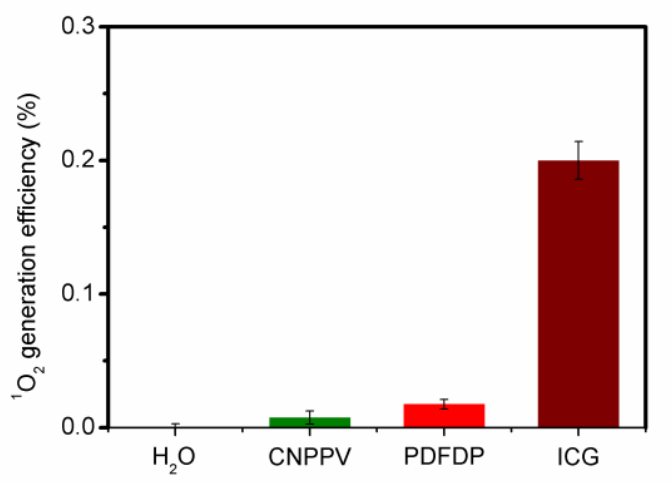

Figure S13. Singlet oxygen generation efficiency of CNPPV and PDFDP Pdots in aqueous solution. ICG (indocyanine green) has been used as standard for detection of ${ }^{1} \mathrm{O}_{2}$ genration efficiency in vitro. With pure water $(0 \%)$ and ICG $(0.2 \%)$ as negative and positive control groups, the ${ }^{1} \mathrm{O}_{2}$ generation efficiency of CNPPV and PDFDP Pdots are $0.01 \%$ and $0.08 \%$, seperately. We detected ${ }^{1} \mathrm{O}_{2}$ generation efficiency with established protocol according to the literatures ${ }^{[1,2]}$.

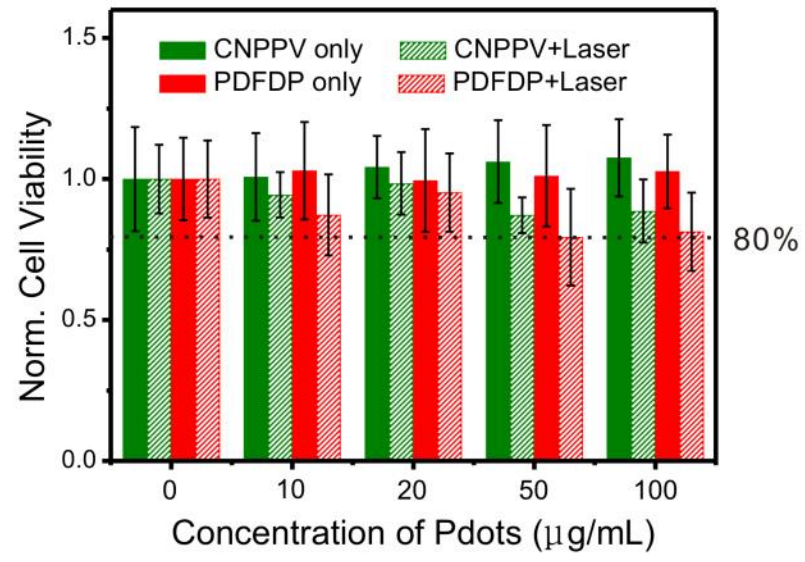

Figure S14. Phototoxicity of Pdots after laser irradiation for 30 minutes.

We incubated CNPPV and PDFDP Pdots (concentrations range from 0 to $100 \mu \mathrm{g} / \mathrm{mL}$ ) with Hela cells overnight, and then irradiated with $760 \mathrm{~nm}$ laser beams $(3 \mathrm{~mW})$ for 30 minutes. The groups without irradiation were set as control. Cell Counting Kit-8 based cell viability assay was performed to check the phototoxicity of Pdots. We detected cell viability according to published literature ${ }^{[3]}$. 

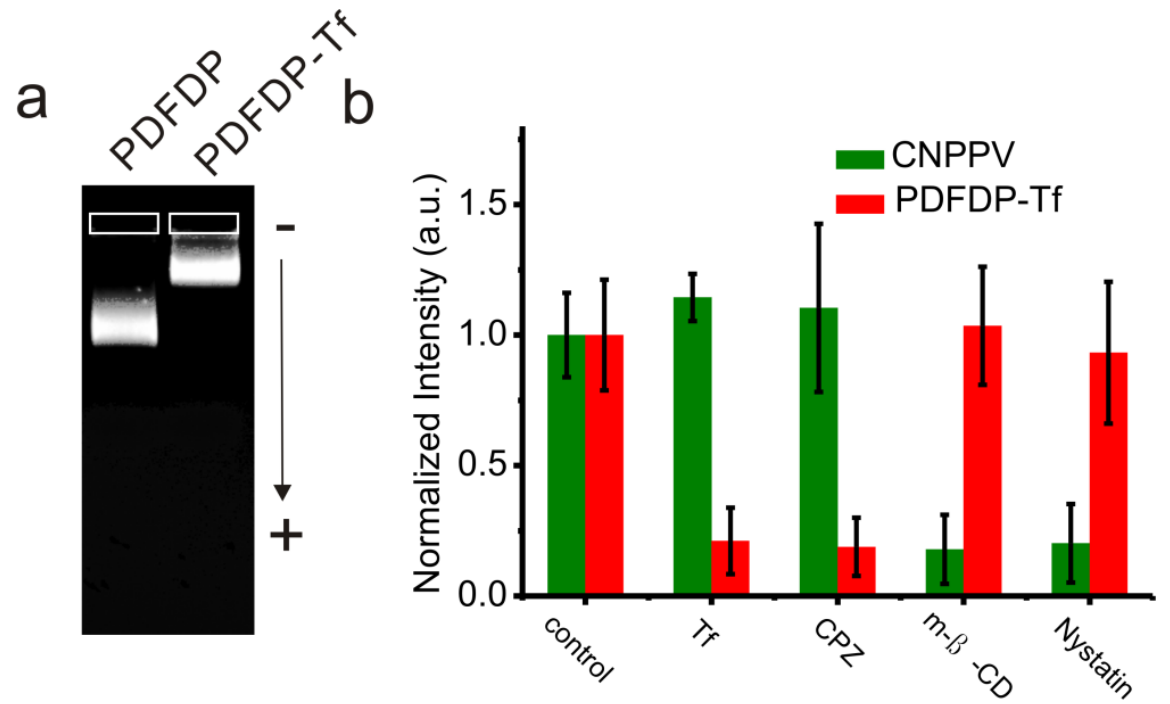

C
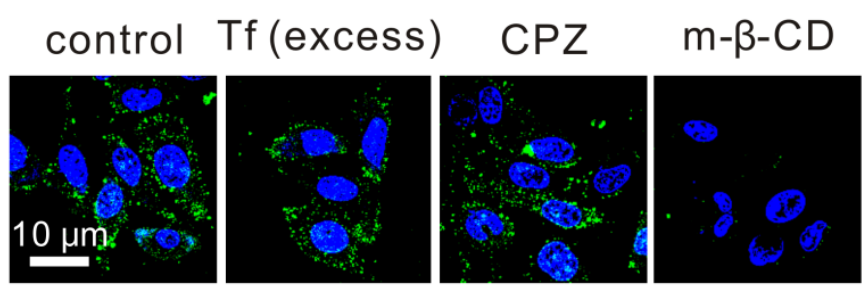

Nystatin
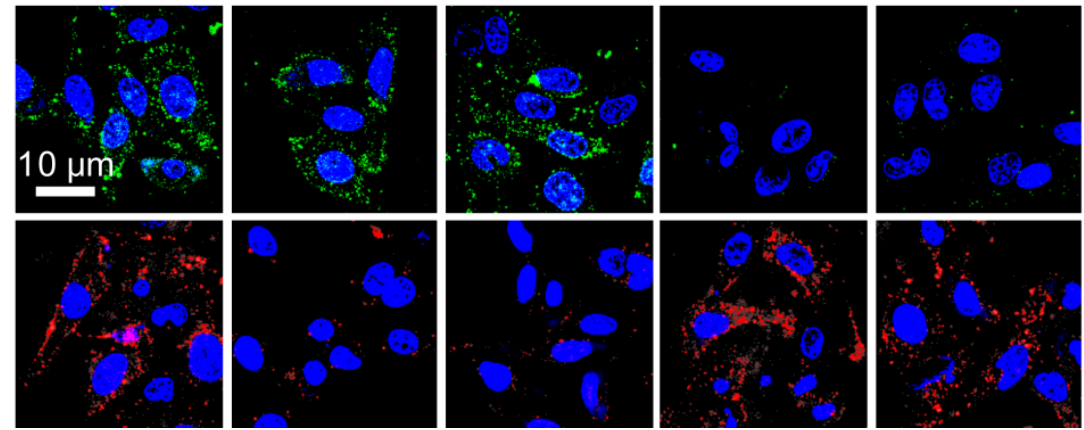

Figure S15. (a) Gel electrophoresis of PDFDP Pdots before and after conjugation with transferrin (Tf). (b) Endocytosis pathway determination for the CNPPV and PDFDP-Tf Pdots. After inhibiting clathrin-mediated endocytosis pathway of Hela cells by excess transferrin or chlorpromazine, uptake of PDFDP-Tf was blocked. The results indicated that PDFDP-Tf Pdots were internalized by clathrin-coated pits. In contrast, CNPPV Pdots were internalized by caveolae, as the uptake was blocked by inhibiting caveolae-mediated endocytosis pathway with m- $\beta-C D$ or Nystatin. (c) Confocal images of CNPPV (upper panel) and PDFDP-Tf (lower panel) Pdots uptake by the Hela cells. Nucleus regions were stained by Hoechst 33258 (blue color). 

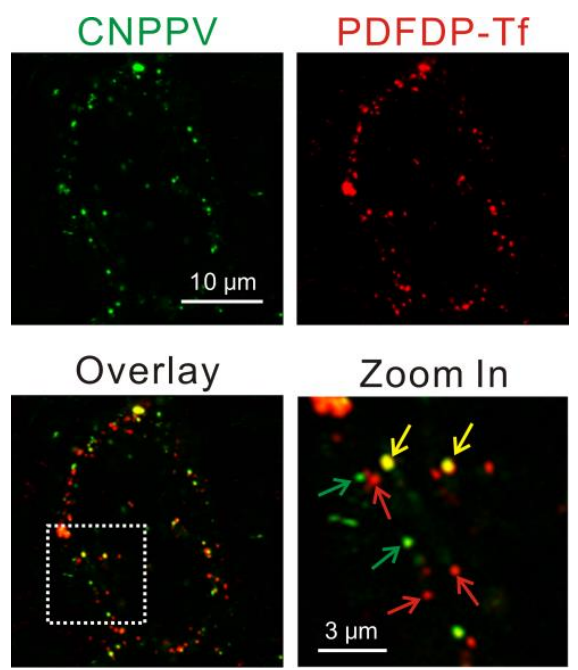

Zoom In

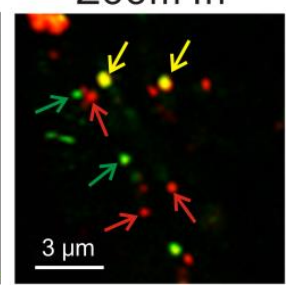

Figure S16. Labeling of clathrin-coated and caveolae vesicles for dual-color confocal imaging. Hela cells were incubated with the mixtures CNPPV and PDFDP-Tf Pdots for specific labeling of caveolae and clathrin-coated vesicles, respectively. As indicated in images, clathrin-coated pits and caveolae were hardly colocalized on cell membrane (CNPPV NPs were indicated by green arrows, PDFDP-Tf NPs were indicated by red arrows). But after endocytosis into cytoplasm, some double-positive vesicles appeared (yellow arrows), indicating the fusion of clathrin-coated and caveolae vesicles in cytoplasm. The observation of the fused clathrin-coated and caveolae vesicles was quite consistent with previously reported work ${ }^{31}$.

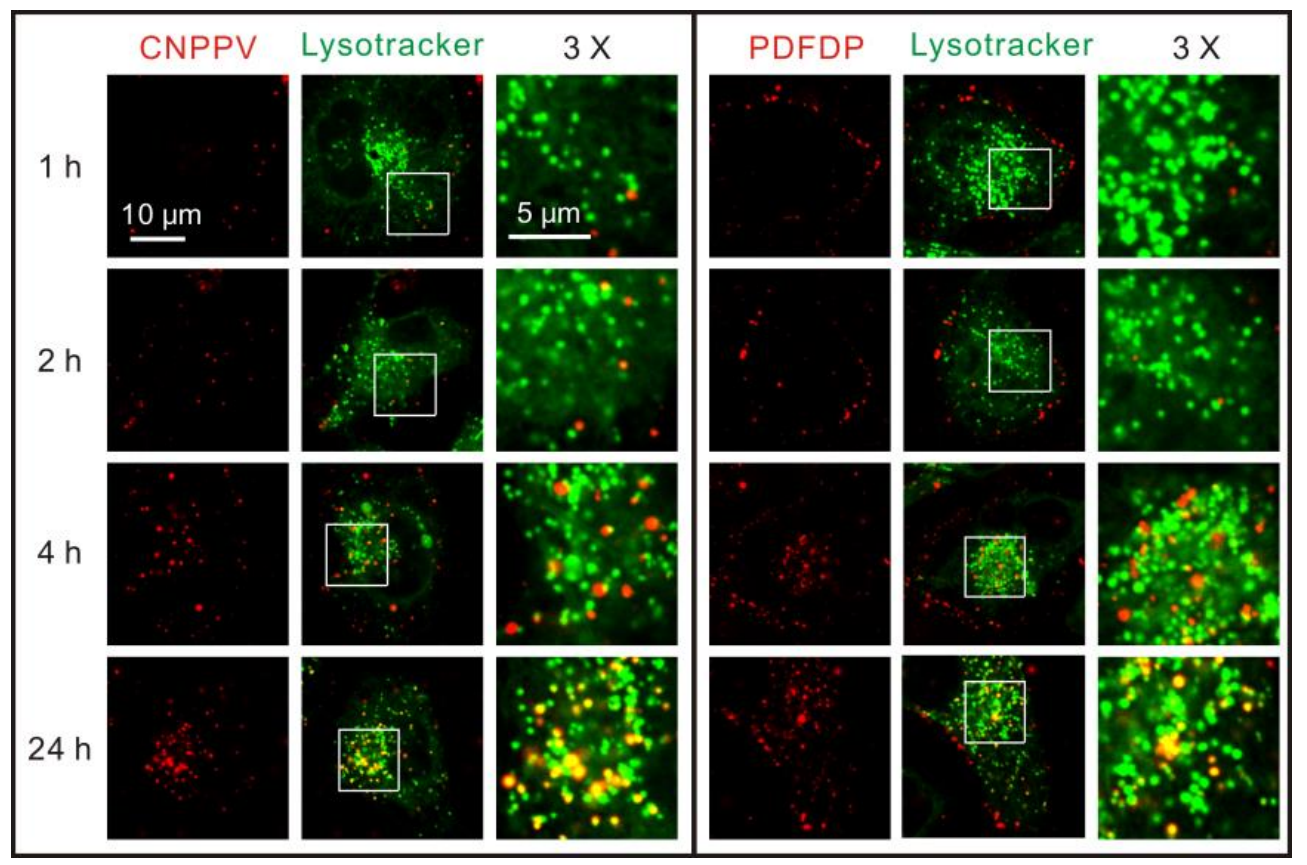

Figure S17. Endocytosis of CNPPV and PDFDP-Tf Pdots. Pdots were internalized into cells through endosomes and located in lysosomes after 24 hours. Within 4 hours after incubation, Pdots were located in endosomes but not in lysosomes. So endosomes were labeled by Pdots within 4 hours. 

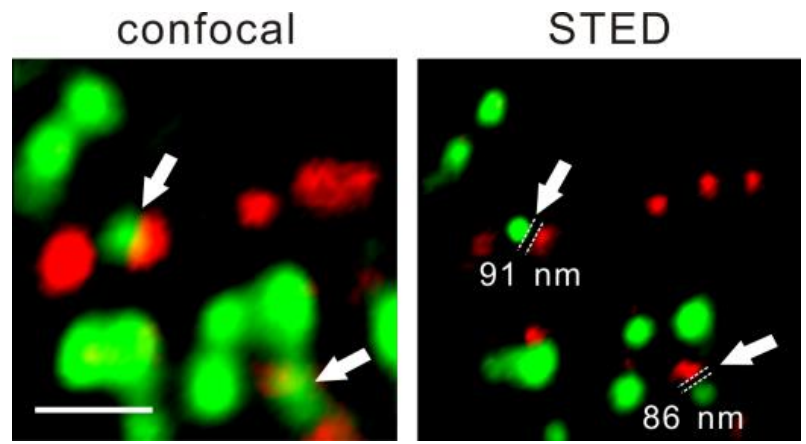

Figure 18: Dual-color STED imaging of endosomes labeled by CNPPV and PDFDP-Tf Pdots. Two neiboring endosmes indicated by white arrows can be easily distignuished in STED mode. As indicated, the distances of two endosomes were $91 \mathrm{~nm}$ and $86 \mathrm{~nm}$, seperately.

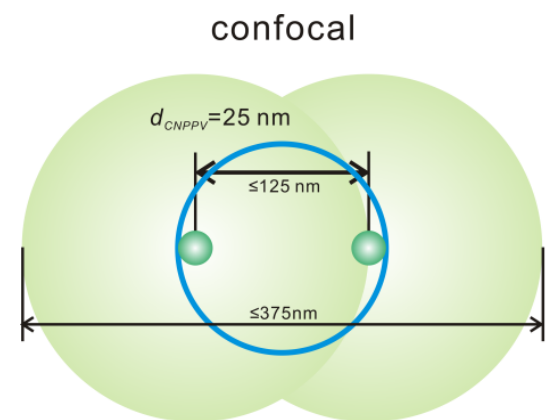

\section{STED}

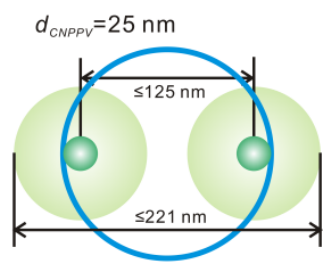

Figure S19. Imaging CNPPV Pdots in one vesicle should have a resolution of less than $375 \mathrm{~nm}$ with confocal microscopy and $221 \mathrm{~nm}$ in STED microscopy. The mean size of a vesicle (blue circle) was estimated to be nearly $150 \mathrm{~nm}$.

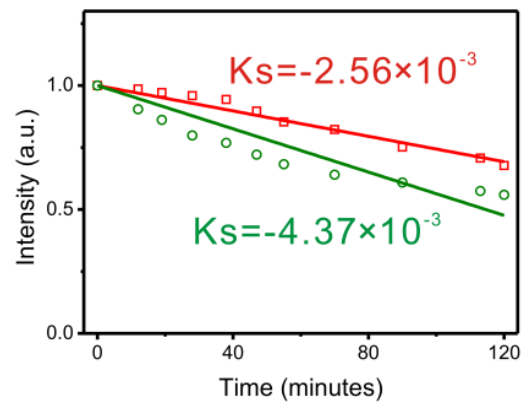

Figure S20. Slop of long-term photobleaching of CNPPV Pdots (green) and PDFDP Pdots (red) after fitting. 

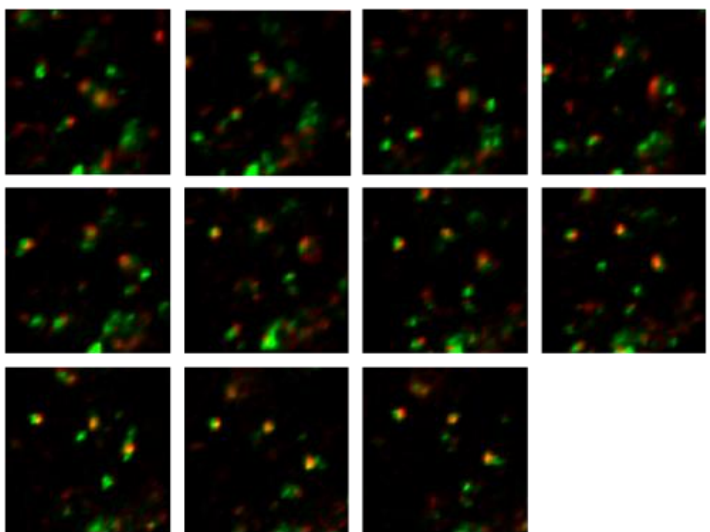

Figure S21. Raw data of figure $5 c$ in the manuscript.

a
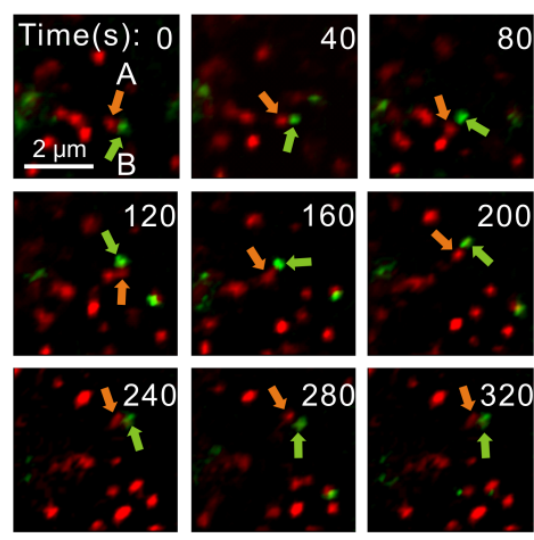

b

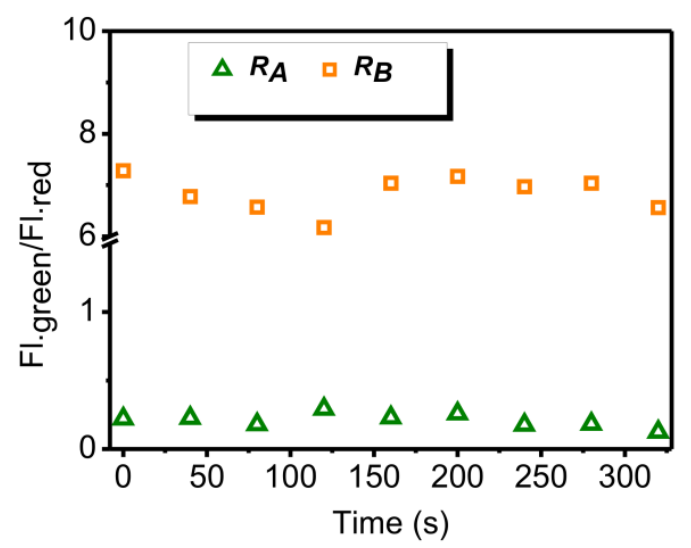

C
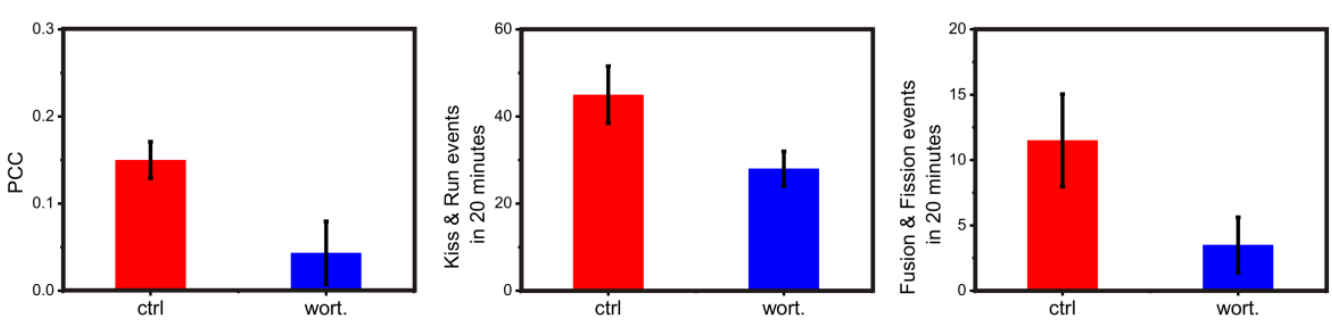

Figure S22. (a) After inhibiting endosome interaction with wortmannin, cargo exchange of two closely located endosomes (endosome A: orange arrow, endosome B: green arrow) were limited. (b) Colorimetric ratio values $\left(R, \mathrm{Fl}_{\text {.green }} / \mathrm{Fl}\right.$.red) of endosome $\mathrm{A}$ ( $R_{\mathrm{A}}$ : green triangle) and $\mathrm{B}$ ( $R_{B}$ : orange square) in 320 seconds in (a). (c) Cells were prelabeled with CNPPV and PDFDP-Tf Pdots. After treated with wortmannin, Pearson's colocalization coefficient (PCC) of cells obviously decreased. "kiss-and-run" (Kiss \& Run) and "fusion and fission" (Fusion \& Fission) events were also reduced in 20 minutes. 


\section{References:}

1. Liang Gao, Ru Liu, Fuping Gao, Yaling Wang, Xinglu Jiang, Xueyun Gao. Plasmon-Mediated Generation of Reactive Oxygen Species from Near-Infrared Light Excited Gold Nanocages for Photodynamic Therapy in Vitro. ACS Nano 2014, 8, 7260-7271;

2. Jingchao Li, Xu Zhen, Yan Lyu, Yuyan Jiang, Jiaguo Huang, Kanyi Pu. Cell Membrane Coated Semiconducting Polymer Nanoparticles for Enhanced Multimodal Cancer Phototheranostics. ACS Nano 2018, 12, 8520-8530.

3. Wu, Y.; Ruan, H.; Zhao, R.; Dong, Z.; Li, W.; Tang, X.; Yuan, J.; Fang, X. Ultrastable Fluorescent Polymer Dots for Stimulated Emission Depletion Bioimaging. Adv. Opt. Mater. 2018, 6, 1800333. 